Revue internationale P.M.E.

Économie et gestion de la petite et moyenne entreprise

\title{
Trajectoires technologiques et mobilisation de réseaux dans des PME de biotechnologie en France et en Grande-Bretagne
}

\section{Jacqueline Estades et Shyama V. Ramani}

Volume 10, numéro 3-4, 1997

URI : https://id.erudit.org/iderudit/1009029ar

DOI : https://doi.org/10.7202/1009029ar

Aller au sommaire du numéro

Éditeur(s)

Presses de l’Université du Québec

ISSN

0776-5436 (imprimé)

1918-9699 (numérique)

Découvrir la revue

Citer cet article

Estades, J. \& Ramani, S. V. (1997). Trajectoires technologiques et mobilisation de réseaux dans des PME de biotechnologie en France et en Grande-Bretagne. Revue internationale P.M.E., 10(3-4), 39-56. https://doi.org/10.7202/1009029ar
Résumé de l'article

L 'objectif de cet article est d1étudier comment des PME en biotechnologie font appel à des réseaux dans la mise en oeuvre d'une trajectoire technologique selon trois options stratégiques : la focalisation sur un segment de la technologie initiale, l'approfondissement de cette technologie ou son élargissement vers des applications diversifiées. En France, ce sont les liens avec les acteurs financiers et politiques qui sont déterminants, alors qu'en Grande-Bretagne, c'est le cas pour les liens avec les grandes entreprises. Les réseaux sont donc une composante majeure de la structure de gouvernance des PME. 


\title{
Trajectoires technologiques et mobilisation de réseaux dans des PME de biotechnologie en France et en Grande-Bretagne
}

\author{
Jacqueline ESTADES \\ Shyama V. RAMANI \\ INRA-SERD, Université Pierre Mendès France
}

MOTS CLÉS

Biotechnologie - PME - Réseaux - Trajectoire technologique Innovation - Alliance stratégique-Coopération

\begin{abstract}
RÉSUMÉ
L'objectif de cet article est d'étudier comment des PME en biotechnologie font appel à des réseaux dans la mise en œuvre d'une trajectoire technologique selon trois options stratégiques: la focalisation sur un segment de la technologie initiale, l'approfondissement de cette technologie ou son élargissement vers des applications
\end{abstract}

\section{LES AUTEURS}

Jacqueline Estades est titulaire d'un DESS et d'un DEA en sociologie de l'apprentissage. Elle enseigne la sociologie des organisations et la sociologie de l'innovation à l'ENESAD de Dijon. Elle est chercheur associé à l'équipe SERD du Laboratoire INRA de Grenoble et travaille sur la dynamique des dispositifs organisationnels liés à la production et à la diffusion des innovations technologiques et plus particulièrement sur les formes de coordination à l'œuvre dans ces dispositifs.

J. Estades, P.B. Joly et V. Mangematin (1996), « Dynamique des relations industrielles dans les laboratoires d'un grand organisme public de recherche: coordination, apprentissage, réputation et confiance ", Sociologie du travail, $n^{\circ} 3 / 96$, p. 391-408.

Shyama V. Ramani est docteur en théorie économique de l'Université Cornell (ÉtatsUnis). Elle est chercheur dans l'équipe SERD-INRA. Elle consacre ses recherches à l'organisation industrielle et à l'économie du changement technique avec, comme champ d'application, les secteurs des biotechnologies à l'échelle mondiale.

D. Jolly et S.V. Ramani, (1996), « Technology creation in the biotechnology sectors: the French connection ", International Journal of Technology Management, Special Issue on Access to Technological and Financial Resources for SME Innovation, vol. 12, n 7/8, p. 830-848.

S.V. Ramani (1996), "Creating incentives: lessons from the French experience", Biotechnology and Development Monitor, $n^{\circ} 26$, p. 18-20. 
diversifiées. En France, ce sont les liens avec les acteurs financiers et politiques qui sont déterminants, alors qu'en Grande-Bretagne, c'est le cas pour les liens avec les grandes entreprises. Les réseaux sont donc une composante majeure de la structure de gouvernance des PME.

\section{ABSTRACT}

The objective of this paper is to study how small and medium-sized biotechnology firms (SMFs) have activated and utilized networks in the course of implementing one of three types of technology strategies : focalization, deepening or widening of the initial technology of the firm. In France, it is the networks with the financial and political actors that are crucial while in Great Britain it is the networks with the large firms that are the most influential. It is shown that networks are a major component of the structure of governance of the SMFs.

\section{RESUMEN}

El objetivo de este articulo consiste en estudiar como las pequeñas y medias empresas can activado y utilizado redes en el curso de la implementación de tres tipos de estrategiás tecnológicas : focalización, ahondamiento, o el alarmamiento de la tecnologia inicial de la empresa. En Francia, son las redes con los actores financieros y politicos que son determinantes, mientras que en Gran Bretaña son las redes con las grandes empresas. Por la tanto, las redes son uno de los más importante componentes de la estructura que gobierna las pequeñas y medias empresas.

\section{Introduction}

Le développement du génie génétique et les percées de la biologie moléculaire ont engendré des innovations technologiques et favorisé la création de petites entreprises de biotechnologies par des chercheurs universitaires et industriels. Ces derniers deviennent des entrepreneurs avec l'aide des institutions publiques et de partenaires privés. Ainsi, les PME de biotechnologies jouent un rôle important de transfert de la recherche vers l'industrie. Elles se sont développées en nombre et en capacité de croissance en mobilisant des réseaux d'acteurs hétérogènes et à l'aide d'accords plus ou moins formalisés avec eux. L'enjeu central pour les créateurs de ce type d'entreprises est de parvenir à s'affirmer avec des avantages concurrentiels dans le marché à partir d'une technologie générique. La compétence technologique d'une firme dérive de sa capacité à exploiter ses ressources pour créer des technologies particulières en fonction de ses besoins, compte tenu de son portefeuille technologique et de sa recherche (Hamel et Prahalad, 1990). La valorisation économique d'une technologie générique implique des choix stratégiques qui engagent des ressources internes et externes à la PME. Sa compétence technologique est fortement dépendante de l'évolution de sa base de connaissances (Freeman, 1991). Les réseaux dans lesquels la PME s'insère jouent un rôle central dans la construction et 
l'évolution de cette base de connaissances. Ils constituent une ressource déterminante de sa capacité à transformer ses compétences technologiques en innovations (Powell, Koput et Smith-Doerr, 1995).

L'objectif de ce travail est d'étudier comment des compétences à organiser des ressources relationnelles sont mobilisées par des PME dans la mise en œuvre d'une trajectoire technologique. Nous avons opté pour une approche empirique dans le but de faire ressortir la diversité des trajectoires à partir de cinq types de variables : des variables structurelles, des variables organisationnelles, des variables technologiques, des variables stratégiques et des variables de marché (voir tableau 1 bis en annexe). Notre analyse s'appuie sur les interviews ${ }^{1}$ des managers actuels des PME dont la plupart en sont les créateurs ${ }^{2}$. Notre terrain est constitué d'un échantillon de 10 PME françaises et de 10 PME anglaises dans le secteur des biotechnologies de nouvelle génération ${ }^{3}$. Les PME ont été sélectionnées sur la base d'une diversité de date de création, de technologie initiale et de structure de capital de départ (voir, en annexe, le profil de l'échantillon). Notre démarche part de l'hypothèse que les accords avec différents acteurs du champ économique et institutionnel sont des éléments structurants de la trajectoire technologique des firmes.

Notre cadrage théorique attribue aux réseaux de relations des PME non seulement un statut de ressource critique mais aussi un rôle actif dans la mise en œuvre d'une trajectoire technologique (sections 1 et 2). À partir de leur compétence à activer des réseaux, nous montrons que les PME mettent en œuvre une trajectoire technologique selon trois options stratégiques : la focalisation, l'approfondissement ou l'élargissement (section 3). Certains acteurs du réseau des firmes sont plus importants que d'autres pour la mise en œuvre de ces trajectoires technologiques, en raison de leur impact sur les différentes fonctions et les prises de décision de l'entreprise. On peut donc les considérer comme des acteurs de la gouvernance des entreprises (section 4).

\section{Compétence technologique et base de connaissances}

L'analyse économique s'est davantage intéressée à la trajectoire technologique des grandes entreprises qu'à celle des PME alors que, dans de nombreux secteurs, les innovations technologiques sont le fait de PME. Cela tient en partie à un déficit

1. Nous adressons nos remerciements à Jacqueline Senker (Science Policy Research Unit, University of Sussex) qui a réalisé les interviews dans les PME anglaises.

2. Le guide d'entretien est construit à partir de quatre thèmes : l'historique de la création, les options technologiques, financières et partenariales de son développement, la qualification de ses ressources humaines et les accords de coopération de la PME.

3. Les technologies génériques développées par ces PME sont le séquençage de gènes, le transfert de gènes, le clonage de gènes, la cartographie génétique et les thérapies géniques. 
d'indicateurs quantitatifs disponibles pour en saisir la dynamique, alors que les grands groupes sont bien caractérisés (sommes consacrées à la R-D, nombre de brevets, etc.). Dodgson (1994) insiste sur l'importance pour l'analyse de la stratégie technologique des PME d'indicateurs qualitatifs tels que les interactions entre des réseaux d'acteurs hétérogènes où s'imbriquent des relations formelles et informelles. Comme le souligne Winter (1984), PME et grandes entreprises n'obéissent pas aux mêmes régimes technologiques, mais cela n'hypothèque pas la possibilité de développement de stratégies technologiques dans les PME.

L'accès aux connaissances génériques les plus récentes dans leur domaine d'activité, la capacité d'investir en R-D et la disponibilité de ressources suffisantes constituent autant d'enjeux majeurs pour les PME en biotechnologie. Ainsi, une trajectoire technologique pourra se définir en termes de développement sur le moyen et le long terme de compétences technologiques spécifiques qui distinguent la PME de ses concurrents, compétences qui se traduiront dans ses ressources humaines, ses équipements, sa base de connaissances et son portefeuille technologique. C'est pourquoi la vitesse de réaction d'une PME à accéder, à développer ou à utiliser des technologies est un puissant atout pour sa trajectoire technologique, car elle rend plus efficaces les processus de décision et l'intégration des activités technologiques dans les différentes fonctions de l'entreprise (Rothwell, 1989). La trajectoire technologique d'une PME dépend donc de ses capacités innovatrices à la fois sur le plan technologique et organisationnel, ce qui suppose des incorporations de compétences et des coopérations avec des acteurs externes disposant de ressources complémentaires.

À la suite de Dodgson (1994), on peut décliner le potentiel innovateur d'une PME de biotechnologie dans quatre directions :

- des capacités d'acquisition et d'implantation de technologies par la R-D,

- des capacités d'apprentissage,

- des capacités de coordination interne de toutes les fonctions de l'entreprise par rapport à ses activités technologiques spécifiques,

- des capacités de choix stratégiques en matière de relations partenariales pour ajuster ses objectifs et ses ressources.

Beaucoup de PME de biotechnologie ont développé leurs compétences technologiques à partir d'une innovation dérivée d'une technologie générique produite antérieurement et extérieurement à leur création. Leurs fondateurs proviennent en grande majorité des laboratoires de la recherche industrielle des grandes entreprises et des laboratoires publics. C'est pourquoi les stratégies de R-D et d'investissement des grandes entreprises ainsi que les politiques publiques de recherche sont des éléments majeurs avec lesquels la trajectoire technologique des PME doit composer. Jouer la carte de la complémentarité avec les grands groupes industriels est un facteur très discriminant de croissance de ces entreprises. Les sociétés de capital 
de risque qui participent fréquemment au lancement de ce type d'entreprises considèrent qu'elles doivent nécessairement s'associer à de grands groupes industriels, car l'accès aux marchés finaux n'est pas dans leur vocation (Lucquin, 1995). Bien qu'indispensables, les collaborations externes ne sont pas des garanties substituables aux compétences internes de l'entreprise. Elles en sont plutôt une ressource complémentaire (Mowery et Rosenberg, 1989), mais à condition que la base de connaissances de la PME puisse les évaluer comme telles. L'enrichissement de la base de connaissances des entreprises est fortement soumise à leur capacité d'absorption de savoirs et de savoir-faire. Outre un gain de réputation et de crédibilité, le développement de coopérations avec de grands groupes industriels ou avec des laboratoires publics infléchit les modalités d'apprentissage de la firme et oriente sa trajectoire technologique. Ces coopérations contribuent à accroître la flexibilité d'une entreprise et l'aident à faire face à des situations peu prévisibles dans un environnement hautement concurrentiel comme celui des biotechnologies (Zucker, Darby et Brewer, 1994).

Les biotechnologies, comme secteur industriel, se construisent sur une base de connaissances complexe et dispersée qui contraint la firme à devoir continuellement composer avec de nouveaux dispositifs de production et d'échanges. Le développement des innovations procède de compétences transversales à plusieurs disciplines scientifiques : elles nécessitent une instrumentation, des savoir-faire, des ressources humaines et financières qui ne peuvent être rassemblées dans le seul espace de l'entreprise. C'est pourquoi la pérennité des PME est subordonnée à l'établissement de relations à la fois flexibles et fiables avec des partenaires diversifiés qui se traduiront par des accords formalisés, marchands ou non marchands, et des échanges plus ou moins tacites d'informations et de matériel. Mustar (1995a, b) montre que la viabilité des entreprises de haute technologie dépend de leur insertion dans un réseau ${ }^{4}$ chaîné et convergent d'acteurs couvrant des registres de compétences diversifiés, intégrant science, technologie et marché.

\section{Mobilisation de réseaux : jeux et enjeux des acteurs}

La notion de réseau est intégrée dans notre analyse non seulement pour rendre compte de formes relationnelles, mais aussi comme un mode intermédiaire de coordination entre hiérarchie et marché. Le réseau se distingue du marché et de la

4. Sur 100 PME créées en France par des chercheurs entre 1983 et 1987, P. Mustar montre qu'en 1993, 16 ont disparu; 12 ont été intégrées et 72 ont survécu. Parmi les 16 qui ont cessé leurs activités, 13 n'ont pas réussi à structurer leur activité en développant des réseaux. Parmi les 12 qui ont été intégrées, 7 sont reliées essentiellement, mais fortement, à un réseau scientifique. Par contre, sur les 72 qui continuent, 32 sont au cœur d'un réseau chaîné et convergent d'acteurs connectant science, technologie et marché. 
hiérarchie dans la mesure où les mécanismes de la coordination des acteurs reposent en partie sur la confiance qui se prouve et s'éprouve dans des échanges répétés. Powell (1990) souligne que les réseaux sont bien adaptés aux circonstances où le succès d'une opération est directement lié à l'information la plus utile à un moment précis, c'est-à-dire celle qui a le plus de valeur. Or, c'est rarement celle qui circule dans la chaîne hiérarchique formelle, ni celle qui peut être référée à des indices de prix. C'est plutôt celle qu'on obtient de quelqu'un avec lequel on a engagé des transactions dans le passé et qu'on a trouvé digne de confiance. Le réseau représente une garantie contre la captation de résultats quand aucun contrat ne peut la prévenir (Porter-Liebeskind, Lumerman, Zucker et Brewer, 1995). Ainsi, les réseaux sont non seulement des sources d'information fiables, mais aussi des dispositifs d'apprentissage organisationnel performants. Certains auteurs les considèrent même comme le véritable centre de production de l'innovation qui s'impose comme forme d'organisation supplantant l'intégration verticale (Powell, Koput et Smith-Doerr, 1995).

L'analyse du rôle des réseaux dans la dynamique d'une organisation implique la prise en compte de trois dimensions (Lazega, 1994): une dimension morphologique relative à l'identité des acteurs qui composent le réseau ; une dimension fonctionnelle qui traduit les influences des différents segments du réseau sur les fonctions de l'entreprise (management, R-D, ressources humaines, production, marketing); une dimension stratégique qui révèle les acteurs décisifs du réseau impulsant la trajectoire technologique de la PME.

Le contenu des interviews révèle la présence de cinq types d'acteurs dans le réseau relationnel des PME : des acteurs financiers, des acteurs scientifiques, des acteurs professionnels, des acteurs politiques et des acteurs industriels dont le rôle n'est que financier dans certains cas. Pour caractériser le réseau de chaque PME à partir de l'appartenance des différents acteurs qui le composent, de leur rôle dans le développement de la PME et des types d'accords qui sous-tendent leurs relations, nous avons dégagé une batterie d'indicateurs qualitatifs de l'analyse de contenu des entretiens (voir tableau 1).

La morphologie du réseau des PME (voir tableau 2) fait ressortir deux tendances dans son mode d'organisation : la prédominance de l'accord bilatéral comme forme de lien avec un acteur externe et la structuration des relations autour de deux pôles que sont la recherche publique et les grandes entreprises.

Dans les deux pays, les liens avec la recherche publique sont une ressource décisive pour la création et le développement de la PME. La recherche universitaire et clinique constitue un réservoir de connaissances, de compétences et d'équipements complémentaires. Le partenariat avec la recherche publique remplit donc plusieurs rôles : celui de coproduction de R-D , de vivier de recrutement, de client pour les PME et de filet de sécurité, grâce à des activités d'enseignement et de codirection de thèses. 
TABLEAU 1

Différenciation des acteurs du réseau

\begin{tabular}{ll}
\hline Acteurs & Indicateurs \\
\hline Financiers (FI) & $\begin{array}{l}\text { Présence dans le capital de la PME. } \\
\text { Participation de la recherche publique aux } \\
\text { ressources de la PME sous forme d'aide } \\
\text { matérielle ou de contrats de recherche } \\
\text { et de prestations de service. }\end{array}$ \\
Engagement de la PME dans des associations \\
Professionnels (PRO) & $\begin{array}{l}\text { professionnelles. } \\
\text { Participation de la PME à des comités d'experts } \\
\text { et à des instances gouvernementales. } \\
\text { Politiques (POL) }\end{array}$ \\
Petites et moyennes entreprises (PME) & $\begin{array}{l}\text { avec une autre PME. } \\
\text { Contrats et alliances stratégiques } \\
\text { avec une grande entreprise. }\end{array}$ \\
\hline
\end{tabular}

TABLEAU 2

Morphologie du réseau relationnel des PME

\begin{tabular}{lcc}
\hline Acteurs & France (F) & Grande-Bretagne (GB) \\
\hline Acteurs financiers (Pers.) & $60 \%$ & $60 \%$ \\
Acteurs financiers (CR) & $60 \%$ & $60 \%$ \\
Acteurs financiers (BC) & $30 \%$ & $30 \%$ \\
Acteurs scientifiques (RU) & $100 \%$ & $90 \%$ \\
Acteurs scientifiques (RC) & $70 \%$ & $30 \%$ \\
Associations professionnelles & $50 \%$ & $50 \%$ \\
Institutions politiques & $70 \%$ & $30 \%$ \\
PME & $40 \%$ & $40 \%$ \\
Grandes entreprises & $50 \%$ & $100 \%$ \\
\hline
\end{tabular}

Pers. (personnes, amis, famille); CR (capital-risque); BC (banques et compagnies d'assurances); RU (recherche universitaire); RC (recherche clinique).

L'analyse de la structure du capital des PME fait ressortir une différence essentielle dans la morphologie du réseau des PME françaises et anglaises. L'entrée de grandes entreprises dans le capital, à la création comme lors des ouvertures de capital, est beaucoup plus élevée en Grande-Bretagne qu'en France. Des grands groupes industriels sont présents dans le capital de $20 \%$ des PME françaises alors qu'ils le sont dans $60 \%$ des cas en Grande-Bretagne. Cette différence repose, pour la France, sur deux raisons principales : d'une part, les grandes entreprises nationales préfèrent entrer dans le capital ou racheter des entreprises en démarrage nordaméricaines et, d'autre part, les PME françaises engagent la plupart de leurs relations 
contractuelles avec des organismes publics de recherche. Par contre, toutes les PME anglaises de notre échantillon développent des coopérations avec des groupes multinationaux soit sous forme de contrats bilatéraux de recherche, soit sous forme d'alliances stratégiques. Ce dernier type d'accord place la grande entreprise à la fois comme partenaire financier de la PME par sa présence dans le capital et comme partenaire de ses options technologiques.

L'apport financier du réseau de relations personnelles des managers-créateurs est loin d'être négligeable dans la structure du capital des PME des deux pays. Il intervient dans $60 \%$ des cas étudiés et résulte d'une démarche volontaire de la part des fondateurs des PME afin de limiter l'influence des sociétés de capital de risque. Les fondateurs souhaitent préserver une part d'autonomie de décision face à ces acteurs financiers sans lesquels ils n'auraient pas pu créer leur entreprise, mais qu'ils estiment trop influents dans les phases d'ouverture de capital.

Si l'État joue un rôle régulateur dans le développement des biotechnologies, ses modalités d'intervention sont fort différentes dans les deux pays. Les aides publiques à la création sont plus importantes en France qu'en Grande-Bretagne. La logique anglo-saxonne est plutôt centrée sur une régulation par le marché alors qu'en France domine une logique programmatique, héritée du modèle «colbertiste » (Salomon, 1986). En Grande-Bretagne, les entreprises s'engagent rarement dans les comités nationaux d'experts chargés de conseiller les gouvernements sur des programmes de recherche. En France, les coopérations avec les instances gouvernementales sont un élément important de la stratégie des PME. C'est une attitude cohérente avec le schéma colbertiste de production d'innovation qui investit l'État du pouvoir régulateur des essais et des erreurs face aux sanctions du marché. Ainsi, les PME compensent le déficit de leur partenariat industriel en s'intégrant dans des programmes de recherche financés par l'État ou la CEE. Cela leur procure également des moyens de contrôle du développement du secteur et de veille de la concurrence.

Le mode d'organisation du réseau relationnel des PME ne les place donc pas au centre d'un ensemble coordonné d'acteurs qu'elles contribueraient à connecter. Elles sont plutôt en situation de gestion d'un portefeuille de relations bilatérales avec des acteurs externes dans le cadre de contrats plus ou moins complets. Elles développent des jeux compensatoires entre les deux polarités dominantes de leur réseau. La focalisation sur la polarité publique supplée la faiblesse de la polarité industrielle dans le cas de la France et la densité des relations industrielles des PME anglaises leur confère une plus grande autonomie à l'égard des sociétés de capital de risque.

\section{Focalisation, approfondissement et élargissement: trois types de trajectoires technologiques}

Pour leur développement technologique, les PME planifient leurs relations partenariales en fonction des ressources complémentaires à leurs activités internes. Elles effectuent une grande partie de leur recherche de base dans le cadre de contrats 
avec des partenaires extérieurs. Ces contrats, nécessairement incomplets, prennent deux formes : soit que la PME conserve la maîtrise du programme et effectue les travaux dans sa structure sur la base d'un accord sur les échéances et les résultats escomptés, soit que le contrat suppose une coparticipation du partenaire. Dans tous les cas, la coordination des acteurs se négocie à trois niveaux : le niveau stratégique pour s'accorder sur les objectifs, le niveau organisationnel pour préciser les moyens de la transaction, et le niveau opérationnel pour définir l'usage dans le temps et l'espace des différents actifs (Brousseau, 1993).

Dans l'ensemble des PME étudiées, c'est le mode de l'accord bilatéral qui préside à l'agencement des relations avec des acteurs externes. Il fait intervenir deux types de contrats : celui qui engage les partenaires dans une opération particulière renouvelable ou non, et celui qui se transforme en alliance stratégique avec un industriel qui devient ainsi co-décideur de la stratégie globale de la PME. Il en découle quatre grandes formes d'organisation des activités externes et internes :

- Les entreprises ont des contrats bilatéraux avec la recherche publique. Parallèlement, elles développent à l'interne des produits à partir de leur technologie de base et les commercialisent par des accords de distribution ou la création de filiales.

- Les entreprises vendent à d'autres entreprises des résultats de recherche interne avec licence exclusive.

- Les entreprises codéveloppent des recherches ou des produits avec des partenaires privés et publics dans le cadre de contrats de coopération bilatérale.

- Les PME passent avec d'autres entreprises des alliances stratégiques. Dans cette configuration, la PME vend, avec licence exclusive, tout ou partie de sa R-D et de sa production à un partenaire industriel. Celui-ci devient de ce fait un acteur de la gouvernance de la PME, car il intervient dans son management stratégique (décisions d'investissements, orientations de R-D, abandon d'activités, restructuration, etc.). En contrepartie, la PME y gagne en crédibilité dans le secteur, en maintien de ses compétences et en sécurité financière.

Les contrats dans lesquels les PME sont engagées ont fait évoluer leur technologie de base selon trois types de trajectoire : la focalisation, l'élargissement et l'approfondissement.

- L'approfondissement de la technologie de base se traduit par le maintien des compétences existantes et enrichit la base de connaissances de la PME. Dans notre échantillon, cette option est retenue par $30 \%$ des PME françaises et $40 \%$ des PME anglaises. 
- L'élargissement de la technologie de base vers une diversification des applications implique le développement de procédés et de produits et l'incorporation de compétences nouvelles ; $30 \%$ des PME françaises et $10 \%$ des PME anglaises sont engagées dans cette trajectoire.

- La focalisation conduit les PME à abandonner certaines activités et à recentrer leurs compétences sur le segment de leur technologie où est ciblée une position de niche. Cette orientation prévaut dans $40 \%$ des PME françaises et anglaises.

Les PME anglaises optent essentiellement pour la focalisation ou l'approfondissement dans la mesure où leurs accords avec d'autres entreprises, et en particulier des grands groupes multinationaux, font d'elles des fournisseurs de R-D. Les PME françaises se répartissent dans les trois types de trajectoires, mais dans le cas de la focalisation et de l'approfondissement, leur principal client reste la recherche publique du secteur universitaire et du secteur clinique. Le choix de l'élargissement résulte d'une stratégie de développement interne de produits accompagné d'accords de distribution avec des partenaires commerciaux.

Dans les deux pays, les contrats avec la recherche publique sont une ressource essentielle pour le développement de la base de connaissances des PME. Par contre, la distinction entre les contrats bilatéraux classiques et les alliances stratégiques s'est révélée discriminante dans la trajectoire technologique des PME (voir tableau 3).

TABLEAu 3

Types d'accords et types de partenaires ${ }^{5}$

\begin{tabular}{lcc}
\hline Types d'accords et de partenaires & France (F) & Grande Bretagne (GB) \\
\hline Contrats bilatéraux avec la recherche publique & $100 \%$ & $100 \%$ \\
Contrats bilatéraux avec des PME & $40 \%$ & $0 \%$ \\
Alliances stratégiques avec des PME & $0 \%$ & $40 \%$ \\
Contrats bilatéraux avec des grandes entreprises & $40 \%$ & $60 \%$ \\
Alliances stratégiques avec des grandes entreprises & $20 \%$ & $80 \%$ \\
\hline
\end{tabular}

Dans les PME anglaises, le taux élevé d'alliances stratégiques avec des grandes entreprises oriente les trajectoires technologiques de focalisation et d'approfondissement vers la spécialisation dans un domaine de compétences complémentaires

5. Les pourcentages sont calculés sur la base de la mention par la PME, d'au moins une coopération spécifiant le type de contrat et de partenaire, entre la date de la création et la période de notre enquête. Ainsi, certains contrats bilatéraux n'ont pas été renouvelés et certaines alliances stratégiques ont pris fin. Par exemple, il n'en subsistait aucune en France au moment de notre enquête. 
de celles de leurs partenaires industriels. En France, soit que les PME complètent sur demande les activités de recherche des laboratoires publics ou d'autres industriels par des accords bilatéraux qui les conduisent à la focalisation ou à l'approfondissement, soit qu'elles optent pour l'élargissement des applications de leur technologie initiale et intègrent le marché par des accords de distribution de leurs produits.

\section{Réseau de relations et gouvernance des entreprises}

Les interactions entre la trajectoire technologique d'une PME et ses relations partenariales font bien ressortir le double statut de son réseau qui s'avère à la fois une ressource mobilisable et une contrainte à intégrer dans les décisions, plus particulièrement dans le cas d'accords financiers et d'alliances stratégiques. Ainsi, par exemple, la présence d'un acteur A dans les relations partenariales de la PME peut hypothéquer l'entrée d'un acteur B ou contraindre à l'intégration d'un acteur C. En effet, les objectifs des acteurs du réseau ne sont pas toujours convergents. Les représentations qu'ils ont de l'avenir de la PME ne se réfèrent pas nécessairement aux mêmes normes d'interprétation. Pour s'entendre, il leur faudra trouver un compromis entre leurs « logiques d'action » (Karpik, 1972). Dans le cas de la France, la phase de retrait des sociétés de capital de risque s'est déroulée parfois sur un mode conflictuel entre les chercheurs créateurs de l'entreprise et les acteurs du capital de risque, conflit à propos des compétences managériales requises pour la croissance de ce type de PME et du choix d'actionnaires internationaux devant succéder au capital de risque. Mais, en même temps, cette confrontation est source d'apprentissage pour la firme, car, comme le souligne Reynaud (1989), la plupart des apprentissages se font sous contraintes de moyens ou d'objectifs. Ces apprentissages sont de nature organisationnelle et technologique. En effet, les décisions d'investissement ou les ouvertures de capital peuvent conduire l'entreprise à réorganiser des parties de sa production, à incorporer de nouvelles compétences, à déléguer son marketing à un partenaire industriel, etc. De même, dans le cadre d'alliances stratégiques, l'entreprise peut accroître sa capacité d'intégration d'une circonstance technologique opportune en se focalisant sur une innovation très ciblée qui exclut le maximum de concurrents potentiels.

Les acteurs du réseau des entreprises de notre échantillon se distribuent selon une configuration éclatée en segments cloisonnés qui soulignent l'influence de certains acteurs (voir schémas 1 et 2 ).

Ainsi, les PME françaises sont très dépendantes des acteurs publics (recherche, instances politiques) avec lesquels elles ont noué des relations. On les retrouve en amont et en aval de leur réseau, comme acteurs décisifs à la création de l'entreprise et comme client principal. En Grande-Bretagne, par contre, le mode d'organisation du réseau est différent. $\mathrm{Si}$, en amont, la recherche publique est déterminante pour 
SCHÉmA 1

Configuration du réseau relationnel des PME françaises

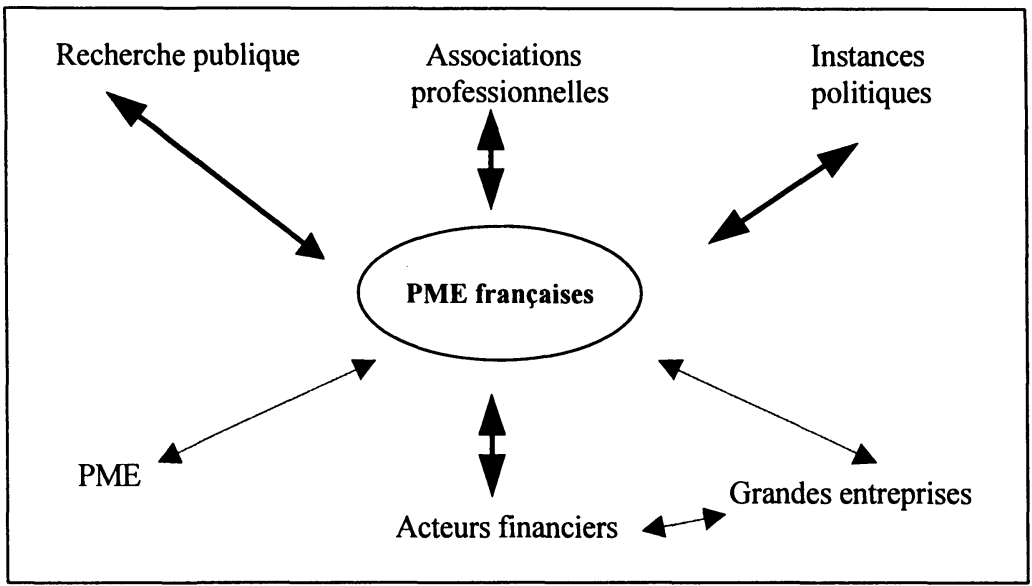

SCHÉma 2

Configuration du réseau relationnel des PME anglaises

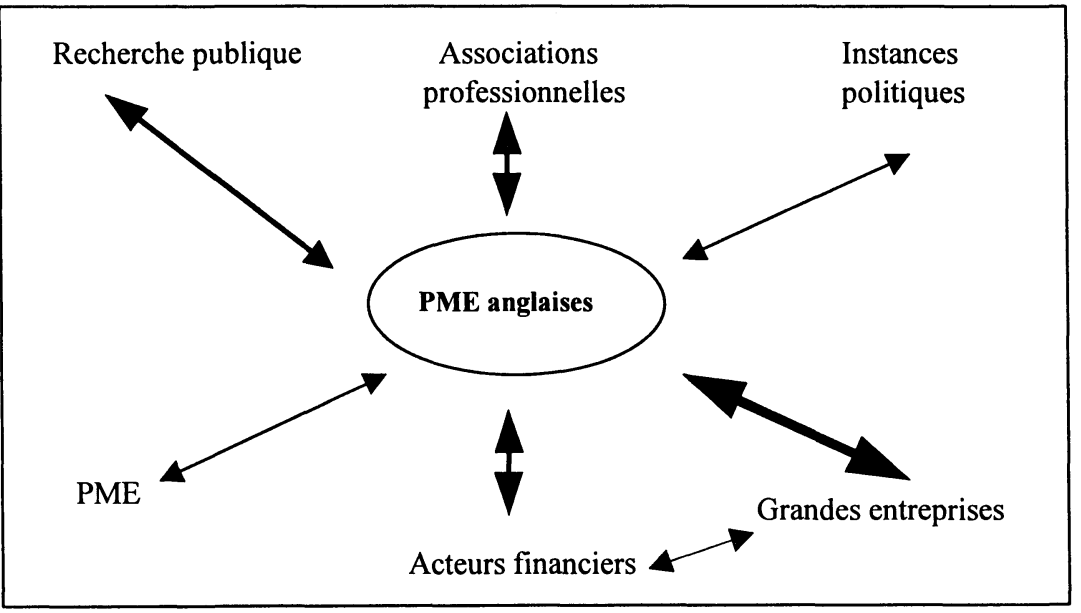


la création des entreprises et l'entretien de leur base de connaissances, celles-ci s'autonomisent en aval des instances publiques et ciblent davantage leurs coopérations dans le secteur industriel.

La croissance des entreprises dépend de l'équilibre entre trois domaines de compétences : le management, la capacité à développer des relations partenariales avec des acteurs scientifiques, financiers et industriels, ainsi que la capacité à affirmer un avantage compétitif sur une technologie générique ou un type de produits. Par rapport à ces enjeux, certains acteurs de la structure de réseau de la PME seront plus décisifs que d'autres lors de prises de décisions majeures visant, par exemple, la levée de capitaux, l'abandon de certaines activités, le développement de nouveaux programmes de recherche ou l'établissement de nouvelles alliances.

Pour identifier les acteurs décisifs pour l'évolution technologique de la PME, nous avons distingué deux périodes : la période de création de l'entreprise $\left(\mathrm{T}^{0}\right)$ et la période (1995) de notre étude ( $\left.\mathrm{T}^{1}\right)$. À partir de l'analyse des entretiens, nous avons dégagé les acteurs qui ont été décisifs en $\mathrm{T}^{0}$ et ceux qui le sont restés ou devenus en $\mathrm{T}^{1}$ (voir tableau 4), et en quoi ils ont infléchi la trajectoire technologique des PME.

TABLEAU 4

Acteurs décisifs et trajectoires technologiques

\begin{tabular}{|c|c|c|c|c|c|c|}
\hline \multirow{2}{*}{$\underbrace{\text { Trajectoires }}_{P M E}{ }^{\text {Périodes }}$} & \multicolumn{2}{|c|}{ APR } & \multicolumn{2}{|c|}{ ELA } & \multicolumn{2}{|c|}{ FOC } \\
\hline & $T^{0}$ & $\mathbf{T}^{\mathbf{1}}$ & $T^{0}$ & $T^{1}$ & $\mathbf{T}^{0}$ & $\mathbf{T}^{1}$ \\
\hline $\begin{array}{l}\text { Acteurs décisifs } \\
\text { en France }\end{array}$ & $\mathrm{SCI}$ & SCI & $\mathrm{SCI}$ & FI & $\mathrm{SCI}$ & $\begin{array}{l}\mathrm{FI} \\
\mathrm{GE}\end{array}$ \\
\hline $\begin{array}{l}\text { Acteurs décisifs } \\
\text { en Grande-Bretagne }\end{array}$ & $\begin{array}{l}\text { SCI } \\
\text { GE }\end{array}$ & GE & SCI & $\mathrm{GE}$ & SCI & FI \\
\hline
\end{tabular}

APR : approfondissement ; ELA : élargissement ; FOC : focalisation ; SCI : acteurs scientifiques ; FI : acteurs financiers; $\mathrm{GE}$ : acteurs grandes entreprises.

À l'origine de la PME, le réseau scientifique s'est avéré une ressource critique dans les deux pays. Il l'est demeuré en France pour la stratégie d'approfondissement, dans la mesure où les partenaires scientifiques de la PME l'ont engagée dans des collaborations technologiques complémentaires de leurs recherches fondamentales sur des thématiques de long terme. Dans les deux autres types de trajectoires technologiques, ce sont les acteurs financiers et en second lieu les grandes entreprises qui ont incité les PME, soit à la focalisation en visant une position de niche, soit à l'élargissement des applications de leur technologie de base. Par contre, en GrandeBretagne, les acteurs industriels ont pesé de manière décisive sur les trois types de stratégie dans la mesure où les alliances stratégiques concernent autant le développement de la R-D que celui des produits. 
Les réseaux décisifs pour les stratégies technologiques sont donc partie prenante de la gouvernance des entreprises en intervenant sur le management et les décisions de coopération. Pour identifier ces influences, nous avons retenu trois types d'indicateurs : la permanence ou le changement de manager, la R-D effectuée ou non avec de grandes entreprises (GE) et le développement interne ou le codéveloppement de produits avec des grands groupes industriels (voir tableau 5). Dans la mesure où les premiers managers, fondateurs des entreprises, sont issus de la recherche, le premier indicateur vise à voir si l'incorporation de compétences managériales plus axées sur le développement financier et commercial de la PME a été encouragée et par quels acteurs de son réseau partenarial. Les deux autres indicateurs renseignent sur les stratégies d'acquisition de connaissances et de compétences technologiques des PME, ainsi que sur leur rôle dans le développement du secteur des biotechnologies par rapport aux grands groupes industriels du secteur pharmaceutique et chimique.

\section{TABLEAU 5}

Acteurs décisifs et gouvernance des entreprises

\begin{tabular}{|c|c|c|c|c|c|c|}
\hline & \multicolumn{2}{|c|}{ APR } & \multicolumn{2}{|c|}{ ELA } & \multicolumn{2}{|c|}{ FOC } \\
\hline & $\mathrm{F}$ & G-B & $\mathrm{F}$ & G-B & $\mathrm{F}$ & G-B \\
\hline $\begin{array}{l}\text { Taux de changement } \\
\text { des managers }\end{array}$ & non & oui & non & non & oui & oui \\
\hline $\begin{array}{l}\text { R-D avec grandes } \\
\text { entreprises }\end{array}$ & non & oui & non & non & oui & oui \\
\hline $\begin{array}{l}\text { Développement } \\
\text { de produits }\end{array}$ & interne & $\begin{array}{l}\text { codévelop- } \\
\text { pement }\end{array}$ & interne & $\begin{array}{l}\text { codévelop- } \\
\text { pement }\end{array}$ & interne & $\begin{array}{l}\text { codévelop- } \\
\text { pement }\end{array}$ \\
\hline
\end{tabular}

Les entreprises ayant une stratégie de focalisation se caractérisent par une rotation des managers sous l'influence des acteurs financiers ${ }^{6}$ et industriels. Elles combinent R-D interne et externe en partenariat avec de grandes entreprises. Dans les trois types de stratégie, non seulement les PME de Grande-Bretagne codéveloppent des produits avec des industriels, mais elles vendent aussi aux grands groupes chimiques et pharmaceutiques des produits intermédiaires pour leur R-D et leur production. Elles sont fortement tirées vers un marché intermédiaire par leur partenariat industriel très intégré dans le pilotage de l'entreprise par les alliances stratégiques. Par contre, les entreprises françaises ont opté soit pour du développement interne de produits et des accords de distribution, compte tenu de la faiblesse de leur partenariat industriel, soit pour une position de prestataire de la recherche publique.

6. Les acteurs du capital-risque considèrent que le changement de manager dans les trois ans qui suivent la création est indispensable à la croissance de l'entreprise. 


\section{Conclusion}

Les compétences à activer des réseaux et le mode d'organisation de ces réseaux conduisent les PME vers la mise en œuvre de trajectoires technologiques selon trois options stratégiques : la focalisation sur un segment de la technologie initiale, l'approfondissement de la technologie initiale ou l'élargissement vers des applications diversifiées de la technologie initiale. Certains acteurs de la structure de réseau des firmes sont plus décisifs que d'autres dans la mise en œuvre de ces stratégies technologiques en raison de leur impact sur les prises de décisions engageant l'avenir de l'entreprise. Ils sont donc une composante majeure de sa structure de gouvernance. En France, on constate une grande dépendance de la PME à l'égard du secteur public et des instances politiques que l'on retrouve en amont comme financiers et en aval comme clients de la PME. En Grande-Bretagne, on remarque une dissociation plus affirmée entre les acteurs d'amont (recherche scientifique), qui sont à l'origine de l'entreprise, et les acteurs d'aval (les industriels), qui sont les partenaires privilégiés pour le développement de l'entreprise. Mais, quelles que soient leurs stratégies, les PME sont avant tout des fournisseurs de technologies pour la recherche publique et d'autres entreprises.

Les tendances observées aux États-Unis ont conduit à esquisser un schéma général de développement d'une PME de biotechnologie caractérisé par trois principaux stades : 1) une première phase de R-D où les PME seraient des fournisseurs de connaissances pour les grands groupes grâce aux ressources des contrats de recherche et au financement du capital-risque ; 2) un second stade centré sur le développement de technologies (contrats de licences); 3) un troisième stade axé sur le développement de produits. Mais il n'existe pas de modèle «idéal » de développement d'une PME de biotechnologie avec des étapes que chaque PME devrait nécessairement franchir pour survivre. La diversité des situations étudiées nous amène plutôt à conclure qu'une PME de biotechnologie peut fort bien se développer sans passer par ces trois stades.

\section{Bibliographie}

ADLER, P.S. (1989), «Technology strategy. A guide to the literature », Research in Technological Innovation, Management and Policy, $\mathrm{n}^{\circ} 4$, p. 25-151.

AKRICH, M. (1989), «La construction d'un système socio-technique : esquisse pour une anthropologie des techniques ", Anthropologie et Sociétés, vol. 13, n 2, p. 31-54.

Brousseau, E. (1993), L'Économie des Contrats. Technologies de l'information et coordination interentreprises, Paris, PUF. 
CALLON, M. (dir.) [1989], La science et ses réseaux : genèse et circulation des faits scientifiques, Paris, La Découverte.

CALlon, M. (1992), «Sociologie des sciences et économie du changement technique : l'irrésistible montée des réseaux socio-techniques », Ces réseaux que la raison ignore, Paris, L'Harmattan, p. 53-78.

DAugeras, B. (1995), «Le rôle du capital de risque », Biofutur, n 150, novembre, p. 26-30.

De Looze, M. A. , J. Estades, P.-B. Joly, S.V. Ramani, P.P. Saviotti, J. Senker et J.L. PEDERSEN (1996), The Role of SMEs in Technology Creation and Diffusion: Implications for European Competitiveness in Biotechnology, Rapport de recherche pour la Commission des Communautés européennes, CT-942032, Grenoble, INRA-SERD.

Dodgson, M. (1994), «La trajectoire technologique dans les PME : quelques particularités et caractéristiques », Revue internationale P.M.E., vol. 7, nos 3-4, p. 201-219.

FREEMAN, C. (1991), « Networks of innovators. A synthesis of research issues », Research Policy, vol. 20, p. 499-514.

GRANOVETTER, M. (1985), «Economic action and social structure : the problem of embeddedness ", American Journal of Sociology, vol. 91, n 3, p. 481-510.

Hamel G. et C.K. Prahalad (1990), «The core competence of a corporation », Harvard Business Review, p. 79-91.

KARPIK, L. (1972), «Les politiques et les logiques d'action de la grande entreprise industrielle» Revue Française de Sociologie, vol. 1-72, p. 82-105.

LAtour, B. (1989), La science en action, Paris, La Découverte.

LAZEGA, E. (1994), «Analyse de réseaux et sociologie des organisations », Revue Française de Sociologie, avril-juin, vol. $35, \mathrm{n}^{\circ} 2$, p. 293-320.

LUCQUiN, D. (1995), «Est-il encore possible de créer des sociétés de biotechnologie en France?», Biofutur, $n^{\circ} 150$, novembre, p. 8-15.

MARCH, J.G. (1991), Décisions et organisations, traduit de l'anglais par M. Waquet, Paris, Éditions d'Organisation.

MERTon, R. (1973), The Sociology of Science, Chicago, University of Chicago Press.

MOWERY, D.C. et N. ROSENBERG (1989), Technology and the Pursuit of Economic Growth, New York, Cambridge University Press.

Mustar, P. (1995a), «Création d'entreprises : bilan de la décennie », Biofutur, nº 150. novembre, p. 20-24.

MuSTAR, P. (1995b), Science et Innovation. Annuaire raisonné de la création d'entreprises par les chercheurs, Paris, Economica. 
Niosi, J., B. Bellon, P.P. SAviotTI et M. Crow (1992), «Les systèmes nationaux d'innovation : à la recherche d'un concept utilisable», Revue Française d'Économie, vol. $7, n^{\circ} 1$, p. $215-250$.

Porter-Liebeskind, J., O.A. Lumerman, L.G. ZuCKer et M.B. Brewer (1995), Social Network Learning and Flexibility: Sourcing Scientific Knowledge in New Biotechnology Firms, Cahier de recherche n o 5320, Cambridge (É.-U.), National Bureau of Economic Research.

POWELL, W. (1990), «Neither market or hierarchy : networks forms of organization », Research in Organizational Behavior, $\mathrm{n}^{\circ}$ 12, p. 295-336.

POWELl, W., K. KOPUT et L. SMITH-DOERR (1995), «Interorganizational collaboration and the locus of innovation : networks of learning in biotechnology », Cahier de recherche, Social and Behavioral Sciences Research Institute, Univ. of Arizona, Aspen Institute Nonprofit Sector Research Fund, College of Business and Public Administration of Arizona.

REYNAUD, J.D. (1989), Les règles du jeu. L'action collective et la régulation sociale, Paris, Armand Colin.

ROTHWELL, R. (1983), «Innovation and firm size : a case of dynamic complementarity ", Journal of General Management, vol. 8, $\mathrm{n}^{\mathrm{0}} 3$, p. 5-25.

Rothwell, R. (1989), "Small firms, innovation and industrial change», Small Business Economic, vol. 1, p. 51-64.

Salomon, J.J. (1986), Le Gaulois, le Cow-boy et le Samouraï : la politique française de la technologie, Paris, Economica.

SwEDBERG, R. (1994), Une histoire de la sociologie économique, Paris, Éd. Desclée de Brouwer.

WALSH, V., J. Niosi et P. MUSTAR (1995), «Small-firm formation in biotechnology : a comparison of France, Britain and Canada ", Technovation, vol. 15, nº 5, p. 303-327.

WINTER, S.G. (1984), "Schumpeterian competition in alternative technological regimes», Journal of Economic Behavior and Organization, vol. 5, p. 287-320.

YoN, B. (1992), Innovation et capital-risque : le cas des biotechnologies, Paris, Éditions d'Organisation.

ZUCKER, L.G. (1986), «Production of trust : institutional sources of economic structure, 1840-1920», Research in Organizational Behavior, vol. 8, p. 53-111.

ZUCKER, L.G., M. DARBY et M. BREWER (1994), «Intellectual capital and the birth of U.S. biotechnology enterprises», Cahier de recherche $n^{0} 4653$, Cambridge (É.-U.), National Bureau of Economic Research. 


\section{Annexe}

\section{TABLEAu 1 bis}

\section{Récapitulatif des variables retenues pour la construction de l'enquête}

Variables structurelles

Variables organisationnelles

Variables technologiques

Variables stratégiques

Variables de marché
Historique de la PME (acteurs, technologie initiale, objectifs, performances).

Management. Évolution des ressources humaines.

Base de connaissances et portefeuille technologique.

Types d'accords et types de partenaires.

Types de clients. Localisation des marchés.

Profil de l'échantillon des entreprises étudiées

TABLEAu 2 bis

Période de création des PME

\begin{tabular}{|c|c|c|c|}
\hline Dates & $\begin{array}{l}1980 \\
1985\end{array}$ & $\begin{array}{l}1985 \\
1990\end{array}$ & $\begin{array}{l}\text { Après } \\
1990\end{array}$ \\
\hline $\mathrm{F}$ & $40 \%$ & $50 \%$ & $10 \%$ \\
\hline G.-B. & $50 \%$ & $30 \%$ & $20 \%$ \\
\hline
\end{tabular}

TABLEAU 3 bis

Provenance des créateurs des PME

\begin{tabular}{lllll}
\hline Dates & RP & RI & RP + RI \\
& Pays & & & \\
\hline F. & $70 \%$ & $10 \%$ & $20 \%$ \\
G.-B. & $60 \%$ & $20 \%$ & $20 \%$ \\
\hline
\end{tabular}

Légende : $R P$ : recherche publique; $R I$ : recherche industrielle; $R P+R I$ : cas d'association entre un chercheur universitaire et un chercheur industriel pour la création d'une PME.

TABlEAU 4 bis

Présence du capital-risque (CR)

dans la structure du capital de la PME à sa création

\begin{tabular}{lccc}
\hline Pays & Aucun & $1 \mathrm{CR}$ & $\begin{array}{c}\text { Plusieurs } \\
\text { CR }\end{array}$ \\
\hline F. & $40 \%$ & $10 \%$ & $50 \%$ \\
G.-B. & $70 \%$ & $10 \%$ & $20 \%$ \\
\hline
\end{tabular}

